\title{
Assessment of genetic diversity in Nordic timothy (Phleum pratense L.)
}

Pirjo Tanhuanpää ${ }^{*}$, Maria Erkkilä2${ }^{2}$, Ruslan Kalendar ${ }^{2}$, Alan Howard Schulman $^{1,3}$ and Outi Manninen ${ }^{4}$

\begin{abstract}
Background: Timothy (Phleum pratense L.), a cool-season hexaploid perennial, is the most important forage grass species in Nordic countries. Earlier analyses of genetic diversity in a collection of 96 genebank accessions of timothy with SSR markers demonstrated high levels of diversity but could not resolve population structure. Therefore, we examined a subset of 51 accessions with REMAP markers, which are based on retrotransposons, and compared the diversity results with those obtained with SSR markers.

Results: Using four primer combinations, 533 REMAP markers were analyzed, compared with 464 polymorphic alleles in the 13 SSR loci previously. The average marker index, which describes information obtained per experiment (per primer combination or locus) was over six times higher with REMAPs. Most of the variation found was within accessions, with somewhat less, $89 \%$, for REMAPs, than for SSR, with $93 \%$.

Conclusions: SSRs revealed differences in the level of diversity slightly better than REMAPs but neither marker type could reveal any clear clustering of accessions based on countries, vegetation zones, or different cultivar types. In our study, reliable evaluation of SSR allele dosages was not possible, so each allele had to be handled as a dominant marker. SSR and REMAP, which report from different mechanisms of generating genetic diversity and from different genomic regions, together indicate a lack of population structure. Taken together, this likely reflects the outcrossing and hexaploid nature of timothy rather than failures of either marker system.
\end{abstract}

Keywords: Genetic diversity, Genetic structure, Phleum pratense L, REMAP, Retrotransposon marker, SSR, Microsatellite, Timothy

\section{Background}

Timothy (Phleum pratense L.), a cool-season perennial, is the most important forage grass species in Nordic countries. Genetic diversity has been previously assessed [1] in a collection of 96 timothy accessions, of which 88 were of Nordic origin. Simple sequence repeat (SSR) markers revealed Nordic timothy accessions to be very polymorphic, having significant differences in the levels of diversity between countries, vegetation zones, and different cultivar types. However, most of the variation (94. \%) existed within accessions, and no clear clustering of accessions based on any grouping was observed. This lack of resolution may either reflect the outcrossing and hexaploid nature of timothy or that SSR markers are not suitable for resolving population structure in timothy.

\footnotetext{
*Correspondence: pirjo.tanhuanpaa@luke.fi

${ }^{1}$ Green Technology, Natural Resources Institute Finland (Luke), Myllytie 1,

FI-31600 Jokioinen, Finland

Full list of author information is available at the end of the article
}

A wide range of DNA markers are available for diversity studies, which all have their advantages and disadvantages. SSRs are amplified from single loci, but are multiallelic and highly polymorphic. Although they are inherited codominantly, separation of different genotypes may not be possible in a polyploid species such as timothy. Therefore, each allele has to be treated as a dominant marker [1]; consequently, the markers that are amplified from the same SSR locus are not independent of each other, and consequentially information is lost. In the REMAP (retrotransposon-microsatellite amplified polymorphism) markers $[2,3]$ assay, the diversity is generated by the integration of retrotransposons, which move in the genome by a copy-and-paste mechanism but are fixed in position upon insertion [4]. They are ubiquitous and abundant in plant genomes, where they are dispersed on all chromosomes [5]. REMAP markers are amplified using a primer designed to a conserved 
retrotransposon region and another anchored to a simple sequence repeat. Products from multiple loci are produced in one PCR reaction, each with only two allele alternatives, a dominant one (amplification) and a recessive one (non-amplification). Because the mechanisms that activate retrotransposons [6] and thereby generate insertional polymorphisms are fully different than that generating SSR allelic variation (polymerase slippage) [7], the two marker systems assay different components of genetic diversity. For potato [8], alfalfa [9], and grapevine [10], retrotransposon and SSR markers in combination were shown to be highly discriminatory and effective.

In the previous study [1], a collection of 96 timothy accessions was analyzed using 13 SSRs, thus describing diversity only at this number of loci. On the other hand, these 13 SSR loci harbored as many as 499 alleles. In the present study, we used REMAP markers for studying diversity in a subset of 51 accessions and compared the results with those obtained with SSR markers. We wanted to determine if another type of marker, which would report from many more locations in the genome and assess different genomic regions where diversity is generated by a different mechanism, could describe diversity more efficiently and also reveal population structure, particularly for a polyploid species. Especially the autonomous nature of retrotransposon diversity generation and display, which is independent of the syntenic organization of polyploids, appeared suited to clonal polyploid species such as timothy. We expected that the retrotransposon markers should thereby be more likely to find genetic structure in timothy, should it exist.

\section{Methods}

\section{Plant material}

In the previous study [1], SSR markers were analyzed in a collection of 96 timothy accessions. Fifty-one of these were selected for the present study to be screened also with REMAP markers (Table 1). Fifteen to twenty randomly selected individuals per accession were investigated, in total 945 individuals. The number of individuals analyzed from each accession in the two studies was not exactly the same because 20 individuals had to be omitted due to their poor amplification in REMAP analysis.

The 51 accessions were mostly wild (30, locations in Fig. 1 in [1]); seven each were classified as landraces, cultivars, and of unknown cultivar types. Accessions were derived from all Scandinavian countries (Denmark, 8; Finland, 10; Iceland, 2; Norway, 10; Sweden, 13). In addition, eight gene bank accessions (so-called exotics) originating from non-Scandinavian countries were included in the study.

\section{Marker analyses}

DNA was extracted using the method of Tinker et al. [11] with some modifications as described in Tanhuanpää and Manninen [1]. Using the iPBS (inter- primer binding site) method, retrotransposon segments were isolated from the timothy genome, sequenced, and long terminal repeats (LTRs) identified [12]. LTR primers were designed to match conserved motifs at or near their termini, according to the methods of Kalendar et al. [13]. For REMAP marker amplification, four different retrotransposon primers (TIM1 - 4) for grasses were used. These were combined with 19 microsatellite-based primers (ISSR + number) that contain repeat units (composed of two or three bases); the 3 ' ends of the primers were anchored by a single nucleotide. Because analyzing markers by gel electrophoresis is very laborious, the retrotransposon primers were labelled with a fluorescent dye, FAM (5-carboxyfluorescein), HEX (hexachloro-6carboxyfluorescein), or TET (6-carboxytetrachlorofluorescein) to enable resolution and visualization of amplification products with a MegaBACE ${ }^{\mathrm{TM}} 500$ Sequencer (GE Healthcare, Buckinghamshire, UK).

Fifty-nine REMAP primer combinations were first tested in a small set of individuals for their functionality and efficiency to produce polymorphic bands. The four best primer combinations were chosen for final analyses (TIM1 with ISSR1, 15 and 20, and TIM2 with ISSR5). These primers, together with their sequences and properties, are shown in Table 2. The REMAP markers were amplified in a reaction volume of $10 \mu$, using $0.25 \mathrm{U}$ of FIREPol $^{\circ}$ DNA polymerase I (Solis BioDyne OU, Tartu, Estonia), buffer B with $2.5 \mathrm{mM} \mathrm{MgCl}{ }_{2}$ as supplied by the enzyme manufacturer, $200 \mu \mathrm{mol} / \mathrm{L}$ each dNTP, $10 \mathrm{ng}$ of DNA, and $500 \mathrm{nmol} / \mathrm{L}$ each primer. The PCR program was run on a PTC-220 DNA Engine Dyad ${ }^{\mathrm{TM}}$ Peltier Thermal Cycler (MJ Research, Waltham, MA, USA) and consisted of an initial denaturation step of 2 min at $94^{\circ}$ C; 32 cycles of $30 \mathrm{~s}$ at $94{ }^{\circ} \mathrm{C}, 30 \mathrm{~s}$ at $60^{\circ} \mathrm{C}$ and $2 \mathrm{~min}$ at $72{ }^{\circ} \mathrm{C}$; a final extension step of $10 \mathrm{~min}$ at $72{ }^{\circ} \mathrm{C}$. After PCR, the amplified products with different labels were combined for MegaBACE runs. SSRs were developed for timothy [14], and analyses were run as described previously [1].

\section{Data analyses}

Each REMAP fragment represents a separate locus, and the presence and absence of the fragment was scored in a binary code $(1 / 0)$. Likewise, each SSR allele was treated as a separate locus and scored in a binary code, even though SSRs are codominant markers. This was because we found the evaluation of allele dosages very unreliable in hexaploid timothy. Diversity indices for markers, including polymorphic information content (PIC), gene diversity, and major allele frequency, were calculated with 
Table 1 Fifty-one Phleum pratense ssp. pratense accessions analyzed in the study

\begin{tabular}{|c|c|c|c|c|c|c|}
\hline Number code & Accession no. & Genebank & Name & Country & Cultivar type $^{1}$ & Veg. zone ${ }^{2}$ \\
\hline 3 & NGB10830 & Nordgen & VA88119 & Denmark & W & 1 \\
\hline 4 & NGB10831 & Nordgen & HF88266 & Denmark & W & 1 \\
\hline 5 & NGB15461 & Nordgen & Vildbjerg AC0103 & Denmark & W & 1 \\
\hline 6 & NGB16650 & Nordgen & Ejsing & Denmark & W & 1 \\
\hline 7 & NGB1672 & Nordgen & BILBO & Denmark & $\mathrm{CV}$ & \\
\hline 8 & NGB1675 & Nordgen & POTA & Denmark & CV & \\
\hline 9 & NGB4053 & Nordgen & SR SALTUM MH0202 & Denmark & W & 1 \\
\hline 10 & NGB4548 & Nordgen & NR FARUP MH0202 & Denmark & W & 1 \\
\hline 11 & NGB132 & Nordgen & LIPINLAHTI ME0901 SEP A & Finland & $\mathrm{L}$ & 4 \\
\hline 13 & NGB14394 & Nordgen & KÄRKÖLÄ HM0102 & Finland & W & 3 \\
\hline 16 & NGB14404 & Nordgen & PAATTINEN MH0201 & Finland & $\mathrm{L}$ & 2 \\
\hline 18 & NGB14417 & Nordgen & MEDVASTÖ MH0101 & Finland & W & 2 \\
\hline 20 & NGB747 & Nordgen & NUWUS AK0401 & Finland & W & 6 \\
\hline 24 & NGB1095 & Nordgen & LAITASAARI ME0201 & Finland & $\mathrm{L}$ & 4 \\
\hline 27 & NGB1111 & Nordgen & MÄLÄSKÄ ME0101 & Finland & $\mathrm{L}$ & 4 \\
\hline 30 & NGB1119 & Nordgen & KATERMA ME0401 & Finland & $\mathrm{L}$ & 4 \\
\hline 32 & NGB2791 & Nordgen & NORRGÅRD AP0101 & Finland & $\mathrm{L}$ & 3 \\
\hline 35 & NGB4066 & Nordgen & TAMMISTO & Finland & CV & \\
\hline 36 & NGB4140 & Nordgen & KORPA & Iceland & $\mathrm{L}$ & \\
\hline 37 & NGB4141 & Nordgen & ADDA & Iceland & CV & \\
\hline 42 & NGB7592 & Nordgen & SKJØLSVIK 01-5-46-5 & Norway & W & 3 \\
\hline 45 & NGB10785 & Nordgen & SANDBU 01-6-49-4 & Norway & W & 5 \\
\hline 47 & NGB17194 & Nordgen & Ifjord 1-1-2-2 & Norway & W & 5 \\
\hline 48 & NGB17198 & Nordgen & Karasjok 1-1-3-2 & Norway & W & 5 \\
\hline 49 & NGB2169 & Nordgen & BODIN & Norway & $\mathrm{CV}$ & \\
\hline 51 & NGB2180 & Nordgen & GRINDSTAD & Norway & $\mathrm{CV}$ & \\
\hline 53 & NGB2918 & Nordgen & HUSETER 01-9-70-1 & Norway & W & 2 \\
\hline 57 & NGB4226 & Nordgen & HATLESTAD 01-7-56-3 & Norway & W & 5 \\
\hline 59 & NGB4231 & Nordgen & GJERDÅKER 01-7-58-1 & Norway & W & 5 \\
\hline 62 & NGB7548 & Nordgen & NAMSVATN 01-5-40-1 & Norway & W & 5 \\
\hline 64 & NGB722 & Nordgen & KUOSSENJARKA JP0404 & Sweden & W & 5 \\
\hline 65 & NGB728 & Nordgen & PJESKER PH0405 & Sweden & W & 4 \\
\hline 66 & NGB11428 & Nordgen & JONATHAN & Sweden & $\mathrm{CV}$ & \\
\hline 69 & NGB14224 & Nordgen & SÖNDRARP IB0101 & Sweden & W & 2 \\
\hline 71 & NGB731 & Nordgen & RÖRMYRBERG JP0204 & Sweden & W & 4 \\
\hline 73 & NGB16975 & Nordgen & NORRA KYLSÄTER FO0103 & Sweden & W & 2 \\
\hline 76 & NGB16981 & Nordgen & BRÄCKETORP FO0501 & Sweden & W & 2 \\
\hline 78 & NGB1306 & Nordgen & BRATTÅKER GB0101 & Sweden & W & 4 \\
\hline 81 & NGB1327 & Nordgen & HAMMARN PR0401 & Sweden & W & 4 \\
\hline 83 & NGB1331 & Nordgen & VÄSTANSJÖ SH0102 & Sweden & W & 4 \\
\hline 85 & NGB1537 & Nordgen & ESKELHEM TL0104 & Sweden & W & 2 \\
\hline 86 & NGB2530 & Nordgen & RÄMNE GJ0301 & Sweden & W & 2 \\
\hline 87 & NGB4349 & Nordgen & BENESTAD JK1506 & Sweden & W & 1 \\
\hline
\end{tabular}


Table 1 Fifty-one Phleum pratense ssp. pratense accessions analyzed in the study (Continued)

\begin{tabular}{lllll}
\hline 89 & PI381926 & GRIN & France & $P$ \\
90 & PI406317 & GRIN & Russia & $P$ \\
91 & IHAR151908 & IHAR & Germany & $P$ \\
92 & PI210426 & GRIN & Greece & $P$ \\
93 & PI325461 & GRIN & Russia & $P$ \\
94 & PI204480 & GRIN & Turkey & $P$ \\
95 & 14G2400116 & RICP & Czech Republic & P \\
96 & RCAT040682 & RCAT & Hungary & W \\
\hline
\end{tabular}

${ }^{1} C V$ advanced cultivar, $L$ traditional cultivar, landrace, $P$ pending, unknown cultivar type, $W$ wild population, weedy

${ }^{2}$ vegetation zones, according to [21]

the program Powermarker v3.0 [15]. A marker index (MI) for each REMAP primer combination and each SSR locus was determined by multiplying the number of polymorphic markers generated $(\mathrm{EMF}=$ Effective multiplex ratio) by average PIC value [16]. It illustrates the amount of information obtained per experiment (per primer combination or locus).

Genetic diversity in each accession was described with five different diversity indices: 1) the number of all markers observed $\left(A_{A}\right)$, corrected to a sample size of $\mathrm{n}=15$ with 1000 resamplings without replacement; 2) the mean number of all markers observed in each individual $\left(A_{I}\right)$; 3) the mean number of pairwise differences (PWD) (Euclidean distances) between individuals, which was counted with the program ARLEQUIN version 2.000 [17]; 4) Shannon's diversity index $I[18]$; 5) the percentage of polymorphic loci. The last two were calculated using the program GenAlex 6.4 [19, 20]. Correlations between diversity indices based on REMAP and SSR markers, and differences in the level of diversity between different

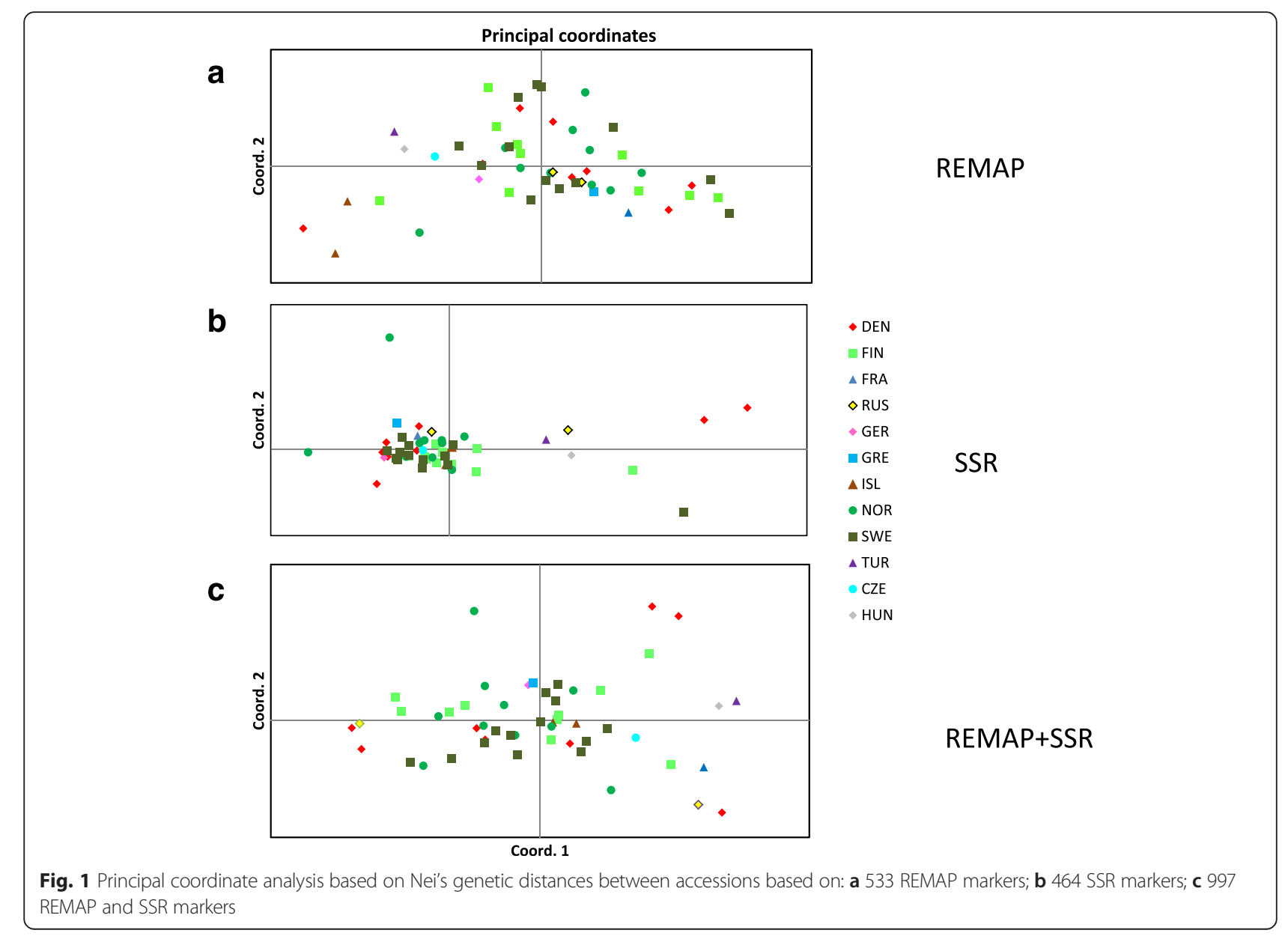


Table 2 REMAP primers that were used in the analysis of timothy diversity, their sequences and properties

\begin{tabular}{|c|c|c|c|c|c|}
\hline Name & Sequence & $\mathrm{nt}$ & $\operatorname{Tm}\left({ }^{\circ} \mathrm{C}\right)$ & CG \% & $\begin{array}{l}\text { Linguistic } \\
\text { complexity (\%) }\end{array}$ \\
\hline TIM1 & GGTGCCGGCATCGATCCTTICA & 22 & 62.4 & 59.1 & 88 \\
\hline TIM2 & ACGAGTGAGGACAAAGTGCGCAGA & 24 & 61.9 & 54.2 & 79 \\
\hline ISSR1 & ACCACCACCACCACCACCC & 19 & 63.2 & 68.4 & 24 \\
\hline ISSR5 & AGCAGCAGCAGCAGCAGCG & 19 & 64.4 & 68.4 & 30 \\
\hline ISSR15 & GTGGTGGTGGTGGTGGTGGTGA & 22 & 64.2 & 63.6 & 28 \\
\hline ISSR20 & TGCTGCTGCTGCTGCTGCC & 19 & 64.6 & 68.4 & 30 \\
\hline
\end{tabular}

nt nucleotides, $T m$ melting temperature, $C G \%$ percentage of $C$ and $\mathrm{G}$ bases

groups such as countries, vegetation zones [21], or cultivar types were determined by ANOVA Proc GLM (SAS Enterprise Guide 4.3).

The program GenAlex $6.4[19,20]$ was used to perform analysis of molecular variance (AMOVA) [22] which partitions total genetic variation to within- and among-accession variance components. The significance of the results was tested by permuting the data 999 times. Principal coordinate analyses (PCA) based on Nei's genetic distances [23] between accessions, and a Mantel test [24], which was used to compare Nei's distances based on REMAP or SSR data, were carried out with the software GenAlex.

\section{Results}

\section{Diversity at marker loci}

Four REMAP primer combinations were used for studying diversity of the 51 accessions. Because not all fragments could be read as marker peaks, selections were made on the basis of the size and shape of the peaks. The numbers of scored polymorphic markers produced by different primer combinations were as follows: TIM2 + ISSR5， 91; TIM1 + ISSR20， 84; TIM1 + ISSR1， 209; TIM1 + ISSR15, 149. A total of 533 REMAP markers were analyzed, ranging in size from 80 to $650 \mathrm{bp}$. A total of 464 polymorphic alleles in the 13 SSR loci were amplified from the 51 accessions, the number varying from 13 to 71 per accession [1]. The average diversity indices of REMAP markers were higher than those of SSR markers (Table 3) leading to a six-fold higher MI for REMAPs.

\section{Genetic diversity within accessions}

The observed number of REMAP markers per accession varied from 195 (PL204480) to 352 (NGB1672) (Table 4), and the number of SSR alleles from 95 (NGB10785) to 194 (NGB1111). There was only one private REMAP marker (in accession PL325461), but 43 private SSR alleles were found [1]. Diversity indices of accessions studied with REMAP or SSR markers, respectively, varied as follows: $A_{I}$ from 47.5 (PL204480) to 84.8 (NGB1672) and from 28.4 (NGB10831) to 35.2 (NGB7592); PWD from 53.1 (PL204480) to 100.2 (NGB1672) and from 28.9 (NGB10785) to 44.9 (NGB7592); I from 0.159 (RCAT040682) to 0.280 (NGB1672), with mean of 0.203 \pm 0.029 , and from 0.109 (NGB722) to 0.156 (NGB1111), with mean of $0.138 \pm 0.014$; the percentage of polymorphic loci from $35.8 \%$ (PL204480) to $64.9 \%$ (NGB1672), with a mean of $49.0 \pm 7.3 \%$, and from $19.8 \%$ (NGB10785) to $41.4 \%$ (NGB1095), with a mean of $34.4 \pm 5.1 \%$ (Table 4 ). The $A_{I}$ values based on SSRs changed slightly from the previous results [1] due to exclusion of 20 individuals (see Methods).

The strength of correlation between diversity indices based on REMAP or SSR markers varied depending on the index. No correlation existed in the level of $A_{I}$. PWD and $I$ correlated weakly at $r=0.27(P=0.059)$ and $r=$ $0.25(P=0.073)$, respectively. The number of markers per accession $\left(\mathrm{A}_{\mathrm{A}}\right)$ correlated moderately at $r=0.37(P=$ $0.0075)$ and the percentage of polymorphic loci strongly with $r=0.44 \quad(P=0.0012)$. Nei's genetic distances between accessions based on REMAP and SSR data correlated strongly $(r=0.67, P<0.001)$ with each other.

When studying levels of diversity between countries, vegetation zones, or different cultivar types, we found no significant differences in $\mathrm{A}_{\mathrm{A}}$ and PWD based on REMAP markers (Table 5). On the other hand, statistically significant $(P<0.05)$ differences in $\mathrm{A}_{\mathrm{A}}$ and PWD between different vegetation zones and in $\mathrm{A}_{\mathrm{A}}$ between different

Table 3 Comparison of diversity measures of REMAP and SSR markers in the analysis of 51 timothy accessions

\begin{tabular}{lll}
\hline & REMAP & SSR \\
\hline No. of primer combinations or loci & 4 & 13 \\
Total no. of markers & 533 & 464 \\
$\begin{array}{l}\text { No. of markers per primer } \\
\text { combination or locus = EMF }\end{array}$ & 133.3 & 35.7 \\
PIC, average & & \\
Markers with PIC $>0.1$ & 0.131 & 0.086 \\
Markers with MAF $<0.1$ & $258=48 \%$ & $148=32 \%$ \\
Average gene diversity & $365=68 \%$ & $371=80 \%$ \\
Marker index (MI) = EMF $\times$ PIC & 0.152 & 0.098 \\
\hline
\end{tabular}

${ }^{1}$ effective multiplex ratio 
Table 4 REMAP and SSR diversity in 51 timothy accessions

\begin{tabular}{|c|c|c|c|c|c|c|c|c|c|c|c|c|c|}
\hline \multirow[b]{2}{*}{ Accession } & \multirow[b]{2}{*}{ No. of ind. } & \multicolumn{4}{|c|}{ REMAP (total no. of markers $=533$ ) } & \multirow[b]{2}{*}{$p^{5}$} & \multirow[b]{2}{*}{$\begin{array}{l}\% \text { polymorphic } \\
\text { loci }\end{array}$} & \multicolumn{4}{|c|}{$\underline{\left.\text { SSR (total no. of markers }{ }^{1}=464\right)}$} & \multirow[b]{2}{*}{$p^{5}$} & \multirow[b]{2}{*}{$\begin{array}{l}\% \text { polymorphic } \\
\text { loci }\end{array}$} \\
\hline & & No. markers & $A_{A}^{2}$ & $A_{1}^{3}$ & $\overline{P W D^{4}}$ & & & No. markers & $\mathrm{A}_{\mathrm{A}}^{2}$ & $A_{1}^{3}$ & $\overline{P W D^{4}}$ & & \\
\hline NGB10830 & 19 & 213 & 200.5 & 55.8 & 61.0 & 0.170 & 38.6 & 124 & 116.7 & 28.8 & 30.7 & 0.114 & 26.7 \\
\hline NGB10831 & 18 & 221 & 207.3 & 50.1 & 56.2 & 0.161 & 40.5 & 148 & 137.9 & 28.4 & 32.5 & 0.126 & 31.3 \\
\hline NGB15461 & 19 & 323 & 302.2 & 73.1 & 85.7 & 0.244 & 59.3 & 188 & 171.5 & 33.3 & 44.0 & 0.155 & 40.5 \\
\hline NGB16650 & 18 & 281 & 264.9 & 60.6 & 71.8 & 0.206 & 52.0 & 142 & 132.9 & 31.3 & 36.2 & 0.127 & 30.6 \\
\hline NGB1672 & 19 & 352 & 328.7 & 84.8 & 100.2 & 0.280 & 64.9 & 162 & 149.7 & 31.7 & 40.1 & 0.137 & 34.7 \\
\hline NGB1675 & 20 & 297 & 277.8 & 78.4 & 87.2 & 0.244 & 54.8 & 119 & 110.1 & 30.0 & 32.5 & 0.111 & 25.6 \\
\hline NGB4053 & 18 & 239 & 226.9 & 58.8 & 65.3 & 0.185 & 44.7 & 145 & 135.2 & 30.7 & 36.2 & 0.135 & 31.3 \\
\hline NGB4548 & 19 & 236 & 219.2 & 54.8 & 61.3 & 0.177 & 43.3 & 154 & 139.4 & 30.1 & 35.1 & 0.137 & 33.2 \\
\hline NGB132 & 19 & 313 & 293.0 & 75.6 & 84.7 & 0.242 & 57.2 & 186 & 167.5 & 31.1 & 39.1 & 0.144 & 39.7 \\
\hline NGB14394 & 19 & 276 & 255.6 & 59.3 & 71.2 & 0.204 & 50.8 & 175 & 162.2 & 32.7 & 42.6 & 0.148 & 37.7 \\
\hline NGB14404 & 20 & 295 & 266.0 & 65.7 & 74.5 & 0.214 & 54.6 & 176 & 158.4 & 33.6 & 40.5 & 0.144 & 37.7 \\
\hline NGB14417 & 18 & 213 & 201.4 & 60.7 & 59.4 & 0.166 & 38.8 & 121 & 115.2 & 28.4 & 32.5 & 0.115 & 25.9 \\
\hline NGB747 & 20 & 310 & 285.9 & 75.2 & 81.5 & 0.235 & 56.8 & 144 & 131.4 & 30.3 & 34.7 & 0.126 & 30.8 \\
\hline NGB1095 & 20 & 330 & 300.6 & 72.9 & 85.0 & 0.245 & 61.2 & 192 & 170.2 & 34.2 & 43.1 & 0.153 & 41.4 \\
\hline NGB1111 & 19 & 308 & 289.2 & 64.3 & 78.1 & 0.229 & 56.7 & 194 & 172.7 & 33.8 & 44.3 & 0.156 & 41.2 \\
\hline NGB1119 & 19 & 226 & 210.1 & 52.8 & 58.6 & 0.170 & 41.5 & 189 & 170.9 & 31.9 & 40.5 & 0.149 & 40.7 \\
\hline NGB2791 & 19 & 315 & 294.5 & 67.7 & 80.7 & 0.238 & 58.2 & 186 & 169.8 & 33.3 & 42.5 & 0.152 & 40.1 \\
\hline NGB4066 & 19 & 259 & 239.8 & 53.6 & 65.4 & 0.188 & 47.5 & 180 & 164.5 & 32.7 & 38.6 & 0.149 & 38.4 \\
\hline NGB4140 & 18 & 263 & 248.3 & 55.0 & 70.5 & 0.201 & 49.0 & 193 & 176.2 & 33.4 & 43.9 & 0.160 & 40.7 \\
\hline NGB4141 & 19 & 260 & 246.6 & 66.5 & 71.6 & 0.206 & 48.0 & 117 & 111.2 & 31.3 & 34.6 & 0.118 & 25.0 \\
\hline NGB7592 & 16 & 244 & 239.2 & 64.5 & 68.6 & 0.192 & 44.7 & 182 & 168.4 & 35.2 & 44.9 & 0.155 & 37.1 \\
\hline NGB10785 & 18 & 202 & 192.0 & 67.0 & 59.9 & 0.165 & 36.6 & 95 & 86.8 & 32.7 & 28.9 & 0.092 & 19.8 \\
\hline NGB17194 & 20 & 286 & 260.7 & 62.1 & 72.7 & 0.213 & 52.7 & 166 & 149.5 & 31.4 & 37.2 & 0.134 & 35.8 \\
\hline NGB17198 & 18 & 336 & 313.7 & 70.1 & 85.7 & 0.245 & 61.9 & 179 & 166.4 & 32.4 & 42.5 & 0.150 & 38.6 \\
\hline NGB2169 & 19 & 290 & 269.0 & 62.9 & 76.5 & 0.219 & 53.8 & 167 & 153.4 & 30.3 & 38.8 & 0.140 & 35.8 \\
\hline NGB2180 & 18 & 304 & 284.6 & 67.7 & 77.4 & 0.226 & 56.1 & 185 & 169.2 & 31.2 & 39.5 & 0.146 & 39.7 \\
\hline NGB2918 & 20 & 332 & 305.3 & 73.4 & 88.2 & 0.252 & 61.7 & 164 & 150.2 & 32.1 & 39.0 & 0.140 & 35.3 \\
\hline NGB4226 & 17 & 236 & 228.4 & 64.4 & 67.9 & 0.191 & 43.3 & 158 & 151.6 & 34.9 & 42.2 & 0.142 & 34.1 \\
\hline NGB4231 & 19 & 230 & 216.4 & 56.3 & 63.0 & 0.183 & 42.2 & 156 & 143.6 & 31.3 & 38.4 & 0.137 & 33.6 \\
\hline NGB7548 & 17 & 272 & 260.1 & 61.4 & 70.0 & 0.201 & 49.7 & 150 & 140.8 & 29.8 & 35.4 & 0.131 & 31.9 \\
\hline NGB722 & 19 & 256 & 241.9 & 77.4 & 71.7 & 0.202 & 45.8 & 115 & 108.8 & 29.5 & 32.7 & 0.109 & 24.4 \\
\hline NGB728 & 18 & 265 & 246.3 & 56.7 & 65.5 & 0.196 & 49.5 & 182 & 171.2 & 33.9 & 43.4 & 0.155 & 39.2 \\
\hline NGB11428 & 16 & 231 & 226.2 & 58.9 & 64.4 & 0.180 & 42.0 & 139 & 138 & 34.2 & 39.8 & 0.135 & 29.7 \\
\hline NGB14224 & 19 & 293 & 273.6 & 65.7 & 79.2 & 0.224 & 54.0 & 175 & 158.4 & 33.4 & 42.2 & 0.146 & 36.6 \\
\hline NGB731 & 20 & 311 & 288.5 & 79.8 & 84.6 & 0.241 & 57.0 & 182 & 163.2 & 31.9 & 41.1 & 0.150 & 39.2 \\
\hline NGB16975 & 19 & 258 & 237.0 & 56.2 & 66.4 & 0.189 & 47.5 & 176 & 161.8 & 33.5 & 42.2 & 0.150 & 37.7 \\
\hline NGB16981 & 15 & 245 & 245.0 & 59.2 & 69.6 & 0.193 & 44.7 & 153 & 149.8 & 33.6 & 41.7 & 0.138 & 32.1 \\
\hline NGB1306 & 18 & 298 & 280.5 & 64.0 & 76.0 & 0.224 & 55.0 & 184 & 173.6 & 32.9 & 41.4 & 0.152 & 39.7 \\
\hline NGB1327 & 19 & 284 & 267.1 & 70.6 & 79.8 & 0.225 & 52.2 & 162 & 151.4 & 33.6 & 41.2 & 0.144 & 34.9 \\
\hline NGB1331 & 19 & 223 & 208.5 & 57.1 & 60.7 & 0.172 & 40.3 & 170 & 158.4 & 32.8 & 42.1 & 0.144 & 36.4 \\
\hline NGB1537 & 16 & 296 & 290.1 & 68.2 & 82.4 & 0.231 & 54.6 & 139 & 131.7 & 30.5 & 36.1 & 0.125 & 28.9 \\
\hline NGB2530 & 19 & 235 & 218.0 & 57.9 & 61.8 & 0.177 & 42.8 & 165 & 152.8 & 31.9 & 39.6 & 0.141 & 35.6 \\
\hline
\end{tabular}


Table 4 REMAP and SSR diversity in 51 timothy accessions (Continued)

\begin{tabular}{|c|c|c|c|c|c|c|c|c|c|c|c|c|c|}
\hline NGB4349 & 20 & 267 & 244.0 & 62.7 & 70.7 & 0.203 & 49.3 & 171 & 153.4 & 32.4 & 40.6 & 0.141 & 36.9 \\
\hline PL381926 & 19 & 240 & 222.2 & 67.4 & 66.5 & 0.187 & 43.9 & 131 & 122.3 & 31.1 & 34.6 & 0.125 & 28.0 \\
\hline PL406317 & 19 & 243 & 226.8 & 56.5 & 64.1 & 0.183 & 44.5 & 165 & 151.7 & 31.8 & 38.5 & 0.145 & 35.6 \\
\hline IHAR151908 & 17 & 259 & 249.0 & 60.3 & 66.4 & 0.192 & 48.0 & 150 & 137.4 & 31.1 & 34.8 & 0.126 & 30.6 \\
\hline PL210426 & 17 & 245 & 237.0 & 65.5 & 69.4 & 0.194 & 44.8 & 146 & 138.9 & 31.8 & 38.3 & 0.131 & 30.6 \\
\hline PL325461 & 17 & 233 & 223.4 & 55.5 & 61.2 & 0.175 & 43.2 & 170 & 157.8 & 30.8 & 39.8 & 0.138 & 34.3 \\
\hline PL204480 & 19 & 195 & 182.4 & 47.5 & 53.1 & 0.151 & 35.8 & 158 & 144.3 & 31.6 & 37.6 & 0.133 & 33.6 \\
\hline $14 G 2400116$ & 19 & 234 & 220.2 & 57.1 & 65.8 & 0.185 & 42.8 & 186 & 170.5 & 34.2 & 44.0 & 0.153 & 39.9 \\
\hline RCAT040682 & 20 & 209 & 191.5 & 50.8 & 55.9 & 0.159 & 38.6 & 157 & 143.5 & 30.5 & 39.1 & 0.141 & 33.8 \\
\hline
\end{tabular}

1each SSR allele treated as a separate marker

${ }^{2}$ corrected number of all markers in each accession

${ }^{3}$ mean number of all markers observed in each individual

${ }^{4}$ mean number of pairwise differences (Euclidean distances) between individuals in each accession

${ }^{5}$ Shannon's diversity index

cultivar types were found with SSR markers (Table 5). In the previous study with 96 accessions analyzed with SSR markers, we found significant differences $(P<0.05)$ in levels of diversity in all groups [1]. When the total number of markers was studied on an individual rather than accession level $\left(A_{I}\right)$, significant differences for each grouping and with both marker types were discovered (Table 5). However, these differences explained only a minor fraction of variation between individuals (1 to $5 \%)$.

\section{Genetic divergence between accessions and groups}

AMOVA was performed in order to divide the total genetic variation into three components: variation within accessions, among accessions, and among countries. Most of the variation in the studied material was found within accessions: $89 \%$ when based on REMAP markers, 93 \% when based on SSR markers, and $91 \%$ when based on both marker types (Table 6).

No genetic divergence was observed between vegetation zones or cultivar types either using SSR or REMAP markers or both (AMOVA, $P<0.05$ ), which might be due to the small numbers of members in different classes. However, the same result was obtained with SSR markers when 96 accessions were studied [1]. In PCA analysis as well, no clustering of accessions based on countries, vegetation zones, or cultivar types was seen (Fig. 1). The first two axes respectively explained $44.1 \%$, $45.8 \%$, or $41.1 \%$ of the variation when REMAPs, SSRs, or both marker types were used in the analysis.

\section{Discussion}

Previously, SSR markers revealed timothy to be very diverse both on the individual and accession level when

Table 5 ANOVA tables indicating F-values, significance levels $P$, and $R^{2}$ for comparisons between different groups for their levels of REMAP and SSR diversity

\begin{tabular}{|c|c|c|c|c|c|c|c|c|c|c|}
\hline \multicolumn{2}{|l|}{ REMAP } & \multicolumn{3}{|c|}{ Total no. of markers $\left(A_{A}\right)$} & \multicolumn{3}{|c|}{ No. of pairwise differences (PWD) } & \multicolumn{3}{|c|}{ Number of markers per individual } \\
\hline Diversity index & df & $\mathrm{F}$ & $P$ & $\mathrm{R}^{2}$ & $\mathrm{~F}$ & $P$ & $\mathrm{R}^{2}$ & $\mathrm{~F}$ & $P$ & $\mathrm{R}^{2}$ \\
\hline \multicolumn{11}{|l|}{ Grouping } \\
\hline Accession & 50 & & & & & & & 6.11 & $<0.001$ & 0.25 \\
\hline Country & 5 & 1.73 & 0.147 & 0.16 & 1.49 & 0.210 & 0.14 & 5.08 & $<0.001$ & 0.03 \\
\hline Vegetation zone & 5 & 0.74 & 0.602 & 0.11 & 0.74 & 0.600 & 0.11 & 5.08 & $<0.001$ & 0.04 \\
\hline Cultivar type & 2 & 2.03 & 0.144 & 0.09 & 1.96 & 0.153 & 0.09 & 3.98 & 0.019 & 0.01 \\
\hline SSR & & \multicolumn{3}{|c|}{ Total no. of markers $\left(A_{A}\right)$} & \multicolumn{3}{|c|}{ No. of pairwise differences (PWD) } & \multicolumn{3}{|c|}{ Number of markers per individual } \\
\hline Diversity index & df & $\mathrm{F}$ & $P$ & $\mathrm{R}^{2}$ & $F$ & P & $R^{2}$ & $\mathrm{~F}$ & $P$ & $R^{2}$ \\
\hline \multicolumn{11}{|l|}{ Grouping } \\
\hline Accession & 50 & & & & & & & 3.18 & $<0.001$ & 0.15 \\
\hline Country & 5 & 1.15 & 0.348 & 0.11 & 1.53 & 0.200 & 0.15 & 5.28 & $<0.001$ & 0.03 \\
\hline Vegetation zone & 5 & 3.90 & 0.008 & 0.40 & 3.49 & 0.014 & 0.38 & 6.67 & $<0.001$ & 0.05 \\
\hline Cultivar type & 2 & 4.70 & 0.015 & 0.19 & 1.71 & 0.194 & 0.08 & 4.98 & 0.007 & 0.01 \\
\hline
\end{tabular}


Table 6 Analysis of molecular variance in 51 timothy accessions based on 533 REMAP markers, 464 SSRs or both

\begin{tabular}{|c|c|c|c|c|c|c|c|c|c|c|c|c|c|}
\hline \multirow[b]{2}{*}{ Source } & \multirow[b]{2}{*}{$d f$} & \multicolumn{4}{|c|}{ REMAP } & \multicolumn{4}{|c|}{ SSR } & \multicolumn{4}{|c|}{ REMAP and SSR } \\
\hline & & SS & MS & $\begin{array}{c}\text { Variance } \\
\text { components }\end{array}$ & $\begin{array}{c}\% \\
\text { total }\end{array}$ & SS & MS & $\begin{array}{c}\text { Variance } \\
\text { components }\end{array}$ & $\begin{array}{c}\% \\
\text { total } \\
\end{array}$ & SS & MS & $\begin{array}{c}\text { Variance } \\
\text { components }\end{array}$ & $\begin{array}{c}\% \\
\text { total }\end{array}$ \\
\hline Among countries & 5 & 896.84 & 179.37 & 0.42 & 1 & 574.09 & 114.82 & 0.21 & 1 & 1471.68 & 294.34 & 0.63 & 1 \\
\hline $\begin{array}{l}\text { Among accessions/ } \\
\text { countries }\end{array}$ & 45 & 5181.97 & 115.15 & 4.21 & 10 & 3719.53 & 82.66 & 2.45 & 6 & 8901.50 & 197.81 & 6.66 & 8 \\
\hline Within accessions & 894 & 33312.58 & 37.26 & 37.26 & 89 & 33271.32 & 37.22 & 37.22 & 93 & 66583.90 & 74.48 & 74.48 & 91 \\
\hline Total & 944 & 39391.39 & & 41.89 & 100 & 37564.94 & & 39.88 & 100 & 76957.08 & & 81.77 & 100 \\
\hline Stat & Value & & & & & Value & & & & Value & & & \\
\hline PhiRT & 0.010 & & & & & 0.005 & & & & 0.008 & & & \\
\hline PhiPR & 0.101 & & & & & 0.062 & & & & 0.082 & & & \\
\hline PhiPT & 0.110 & & & & & 0.067 & & & & 0.089 & & & \\
\hline
\end{tabular}

Probability, $\mathrm{P}($ rand $\geq$ data), for PhiRT, PhiPR and PhiPT $=0.001$, and is based on permutation across the full data set

PhiRT $=A C /(W A+A A+A C)=A C /$ TOT

$\mathrm{PhiPR}=\mathrm{AA} /(\mathrm{WA}+\mathrm{AA})$

$\mathrm{PhiPT}=(\mathrm{AA}+\mathrm{AC}) /(\mathrm{WA}+\mathrm{AA}+\mathrm{AC})=(\mathrm{AA}+\mathrm{AC}) / \mathrm{TOT}$

Key: $A C=$ est. var. among countries, $A A=$ est. var. among accessions, $W A=$ est. var. within accessions

studied in a collection of 96 accessions. Because it was impossible with SSRs to resolve any population or geographical structure [1], we here have applied a very different kind of neutral marker, REMAPs, which are based on displaying retrotransposon insertions.

Both REMAPs and SSRs were highly polymorphic. Variation was observed mostly within accessions but with slightly smaller proportion for REMAPs (89\% vs. $93 \%$ ). This difference may be due to the biology of how SSR and retrotransposon polymorphisms are generated. SSRs are generated by replication slippage [7], a process expected to be independent of the environment. In contrast, retrotransposons are known to be activated by both biotic and abiotic stresses [6], conditions which may well be greater in some populations compared with others. Population-level stress would thereby lower the proportion of polymorphism on the individual level and increase it on population or geographic levels.

Diversity indices in accessions were lower for SSR than for REMAP markers. This is likely because SSR markers (i.e., alleles) are not independent of each other; there is a theoretical maximum number of markers that can exist in one individual. If all SSR loci would amplify from all three genomes of Phleum, the maximum number of markers would be 78 (13 loci, 6 alleles in each). However, there is evidence that timothy is an allopolyploid [25]. Allopolyploidy is consistent with our earlier results [1], with some SSR loci found only in one genome whereas others were present in all three. Therefore, the real maximum number of SSR alleles in any one individual lies somewhere between 26 and 78. In the present study, the observed maximum was 45 .

Polyploids represent about $50 \%$ of flowering plants [26]. In polyploids, the problem of lack of independence between SSR loci is particularly a problem, but given a very high number of loci developed from the genome sequences of major crops such as cotton or wheat, chromosome-specific markers can be recovered [27]. For agricultural species without reference genomes such as timothy or for many wild species [28], selection of markers with diploid inheritance can reduce the usable loci to very low numbers.

In contrast to SSRs, no limit exists for the maximum amount of REMAPs in one individual because retrotransposon insertions are independent of each other. Moreover, different retrotransposon families, such as in the hexaploid wheat genomes [29], show different evolutionary histories, enabling discrimination between homeologues. Retrotransposon markers have been deployed effectively for even the highly polyploid sugarcane [30]. Although codominant REMAPs also exist, codominance does not restrict the possibility of co-existence of markers in one individual. The maximum amount for REMAPs observed in one individual in the present study was 121. Correlations between diversity indices based on REMAP or SSR markers were mostly low or moderate because the two marker systems report from different genomic regions where polymorphisms are generated by different processes. On the other hand, even though SSRs could be treated as codominant markers, it has been suggested that large similarities between diversity indices with dominant markers but somewhat lower between dominant markers and SSRs are due to insufficient numbers of analyzed SSR loci [31].

When using markers for measuring distances, PWD between individuals correlated weakly $(r=0.26)$ but genetic distances between accessions strongly $(r=0.67)$ between the two marker types. PWD is based on the 
Euclidean distances between individuals whereas distances between accessions are based on marker frequencies. The same sort of result - poor or nonexistent individual-by-individual correlations but moderate correlation between accessions - was obtained when amplified fragment length polymorphisms (AFLPs), which are comparable to REMAPs by being a multilocus and dominant marker type, and SSRs were compared [32]. In potato, a low correlation of SSR and REMAP markers $(r=$ $0.17)$ in the Mantel's matrix correspondence test was found [8].

Comparing the two marker types, REMAP markers were more cost-efficient. The PCRs of four REMAP primer combinations were made separately, and products from two different combinations with different fluorescent labels were combined for MegaBACE runs. As a consequence, for the whole diversity analysis study (945 samples), 40 PCRs on 96-well microtitre plates were made and analyzed in 20 Megabace runs. A total of 533 polymorphic markers was produced. On the other hand, the 13 SSR loci were multiplexed into 5 PCR reactions and analyzed in 5 MegaBACE runs, requiring in total 50 PCR plates and 50 MegaBACE runs. In addition, some planning and optimization was required in order to multiplex the PCR reactions for the various SSR loci. A total of 464 SSR markers (i.e., alleles) was amplified. Accordingly, more REMAP markers (i.e., loci) were produced with less labor, money, and time. The MI was over six-fold higher with REMAPs, which is not due only to the need to interpret SSR alleles as separate markers but is also typical for markers with an effective multiplex ratio, and has been detected also when AFLPs have been compared with SSRs [16].

Knowledge of genetic variation and relationships between individuals and accessions is essential when conserving and using genetic resources. Evaluation of genetic diversity requires analysis of multiple markers as efficiently as possible. Choosing a suitable marker type, several aspects have to be taken into account, not only expected heterozygosity and marker index, but also technical difficulty, ease of genotyping, cost, and availability. Technically, there were no differences between REMAPs and SSRs and we encountered analysis difficulties with both marker types. All SSR peaks contained some degree of stutter, which complicated the identification of alleles. On the other hand, interpretation of REMAP markers was very slow because several markers were amplified in one PCR reaction and there was a wide variation in peak heights. All peaks could not be analyzed and selections had to be made according to peak heights and frequency. Difficulties in scoring hindered the use of automated analysis programs for both marker types. Regarding availability, there are universal retrotransposon primers that can be used in any species, and primers specific for Graminae also have a vast range of use. Moreover, SSRs have not been developed for every species, and transform rates from one species to another depends on the genetic distance of the taxa [33]. These general conclusions regarding the utility of SSR and retrotransposon markers alone and in combination are consistent with those for four diverse dicot species, distant from the monocot timothy [8-10].

\section{Conclusions}

When diversity in a polyploid species is examined, where the codominant nature of SSRs is of no use, dominant REMAP markers, as analyzed by size on a sequencer, were more cost-efficient. REMAPs also described diversity from a larger segment of the genome compared to the same number of SSR alleles. On the other hand, SSRs detected differences in the level of diversity in different groups better than REMAPs. Furthermore, private SSR alleles were found, making SSRs better for accession identification. Private alleles, however, can be developed from retrotransposon markers using the RBIP (retrotransposon-based insertion polymorphism) and ISBP (insertion site-based polymorphism) methods, which are locus-specific [34]. Genetic distances between accessions were similar with REMAP or SSR markers, but neither marker type could reveal any clear divergence between vegetation zones, cultivar types or countries in the polyploid, very polymorphic and heterozygous timothy species. SSR and REMAP polymorphisms derive from very different mechanisms. Variations in SSR numbers at individual loci derive from polymerase slippage during replication. In contrast, retrotransposon insertions, which can be stress-driven, generate the priming sites for retrotransposon-based marker methods. Given the vastly different numbers of microsatellite and retrotransposon loci queried by the marker systems used, which report from very different genomic regions, the fact that they together show a lack of structure likely reflects the outcrossing and hexaploid nature of timothy rather than failures of either marker system. Both retrotransposons and SSRs, however, are neutral markers; patterns of variation in the gene space of timothy, such as through single nucleotide polymorphism (SNP) genotyping, remain to be explored. These would allow the possibility to evaluate allele dosages, thereby increasing the information embodied in each locus. SNP markers have been used in sugarcane, a complex autopolyploid species, to estimate ploidy level and also the dosage of SNPs [35]. The availability of SNP markers has increased with the invention of the genotyping by sequencing strategy (GBS) [36] and a recent study presents its use to evaluate allele frequencies in populations in an outbreeding species, perennial ryegrass [37]. Such techniques could be applied to timothy as well to study the structure of 
accessions. On the other hand, the importance of using both molecular and phenotypic markers for assessing diversity especially when evaluating adaptive potential has been emphasized in a study where timothy accessions were characterized with SSRs, chloroplast DNA sequences, as well as by morphological and phenological traits [38].

\section{Competing interests}

The authors declare that they have no competing interests.

\section{Authors' contributions}

OM designed the research; PT analyzed the SSR marker data, carried out all data analyses and wrote the manuscript; RK developed the REMAP primers; ME analyzed the REMAP marker data; OM and ME gave comments on the manuscript; AS revised the manuscript. All authors read and approved the final manuscript.

\section{Acknowledgements}

We want to thank Nordgen, Mika Isolahti and Siri Fjellheim for selecting and providing plant material for the study. We appreciate the excellent technical assistance of Marja-Riitta Arajärvi, Kirsti Mäkelä, Ulla Kojonsaari, Sirpa Moisander, and Anneli Virta. The help of Kristiina Antonius, Marjo Serenius, and Miika Tapio in data analyses is also greatly acknowledged. We are grateful for The Ministry of Agriculture and Forestry in Finland, as well as The Nordic Joint Committee for Agricultural Research for their financial support.

\section{Author details}

'Green Technology, Natural Resources Institute Finland (Luke), Myllytie 1, Fl-31600 Jokioinen, Finland. ${ }^{2}$ Internal Expert Services, Natural Resources Institute Finland (Luke), Humppilantie 14, Fl-31600 Jokioinen, Finland. ${ }^{3}$ Luke/ BI Plant Genome Dynamics Laboratory, Institute of Biotechnology, Viikki Biocenter, University of Helsinki, P.O. Box 56, Viikinkaari 1, Fl-00014 Helsinki, Finland. ${ }^{4}$ Boreal Plant Breeding Ltd, Myllytie 10, Fl-31600 Jokioinen, Finland.

Received: 18 December 2015 Accepted: 19 April 2016

Published online: 26 April 2016

\section{References}

1. Tanhuanpää P, Manninen O. High SSR diversity but little differentiation between accessions of Nordic timothy (Phleum pratense L.). Hereditas. 2012;149:114-27.

2. Kalendar R, Grob T, Regina M, Suoniemi A, Schulman A. IRAP and REMAP: two new retrotransposon-based DNA fingerprinting techniques. Theor Appl Genet. 1999:98:704-11.

3. Kalendar R, Schulman AH. IRAP and REMAP for retrotransposon-based genotyping and fingerprinting. Nat protocols. 2006;1:2478-84.

4. Schulman AH. Retrotransposon replication in plants. Curr Opin Virol. 2013;3: 604-14.

5. Schulman AH, Gupta PK, Varshney RK. Organization of retrotransposons and microsatellites in cereal genomes. In: Gupta PK, Varshney RK, editors. Cereal Genomics. Dordrecht: Kluwer Academic Publishers; 2004. p. 83-118.

6. Grandbastien MA. LTR retrotransposons, handy hitchhikers of plant regulation and stress response. Biochim Biophys Acta. 1849;2015:403-16.

7. Kelkar YD, Eckert KA, Chiaromonte F, Makova KD. A matter of life or death: how microsatellites emerge in and vanish from the human genome. Genome Res. 2011;21:2038-48.

8. Sharma V, Nandineni R. Assessment of genetic diversity among Indian potato (Solanum tuberosum L.) collection using microsatellite and retrotransposon based marker systems. Mol Phylogenet Evol. 2014;73:10-7.

9. Mandoulakani BA, Sadigh P, Azizi H, Piri Y, Nasri S, Arzhangh S. Comparative assessment of IRAP, REMAP, ISSR, and SSR markers for evaluation of genetic diversity of alfalfa (Medicago sativa L.). J Agr Sci Tech. 2015;17:999-1010.

10. Villano C, Carputo D, Frusciante L, Santoro X, Aversano R. Use of SSR and retrotransposon-based markers to interpret the population structure of native grapevines from southern Italy. Mol Biotechnol. 2014;56:1011-20.

11. Tinker NA, Fortin MG, Mather DE. Random amplified polymorphic DNA and pedigree relationships in spring barley. Theor Appl Genet. 1993;85:976-84.
12. Kalendar R, Antonius K, Smykal P, Schulman AH. iPBS: A universal method for DNA fingerprinting and retrotransposon isolation. Theor Appl Genet. 2010;121:1419-30

13. Kalendar $R$, Lee $D$, Schulman $A H$. FastPCR software for $P C R$, in silico $P C R$, and oligonucleotide assembly and analysis. Methods Mol Biol. 2014;1116:271-302.

14. Cai HW, Yuyama N, Tamaki H, Yoshizawa A. Isolation and characterization of simple sequence repeat markers in the hexaploid forage grass timothy (Phleum pratense L.). Theor Appl Genet. 2003;107:1337-49.

15. Liu K, Muse SV. Powermarker: integrated analysis environment for genetic marker data. Bioinformatics. 2005;21:2128-9.

16. Powell W, Morgante M, Andre C, Hanafey M, Vogel J, Tingey S, et al. The comparison of RFLP, RAPD, AFLP and SSR (microsatellite) markers for germplasm analysis. Mol Breeding. 1996;2:225-38.

17. Schneider S, Roessli D, Excoffier L. Arlequin: a software for population genetics data analysis. Ver 2.000. Genetics and Biometry Lab, Department of Anthropology, University of Geneva, Switzerland; 2000.

18. Shannon CE. A mathematical theory of communication. Bell System Tech J. 1948;27:379-423 and 623-56.

19. Peakall R, Smouse PE. GENALEX 6: genetic analysis in Excel. Population genetic software for teaching and research. Mol Ecol Notes. 2006;6:288-95.

20. Peakall R, Smouse PE. GenAIEx 6.5: genetic analysis in Excel. Population genetic software for teaching and research - an update. Bioinformatics. 2012;28:2537-9.

21. Moen A. National Atlas of Norway: Vegetation. Hønefoss: Norwegian Mapping Authority; 1999.

22. Excoffier L, Smouse PE, Quattro JM. Analysis of molecular variance inferred from metric distances among DNA haplotypes: application to human mitochondrial DNA restriction sites. Genetics. 1992;131:479-91.

23. Nei M. Genetic distance between populations. Am Nat. 1972;106:283-92.

24. Mantel NA. The detection of disease clustering and a generalized regression approach. Cancer Res. 1967;27:209-20.

25. Stewart AW, Joachimiak AJ, Ellison NW. Phleum. In: Kole C, editor. Wild crop relatives: genomic and breeding resources. Millets and grasses. Springer Berlin Heidelberg; 2011. p. 257-74.

26. Soltis PS, Marchant DB, Van de Peer Y, Soltis DE. Polyploidy and genome evolution in plants. Curr Opin Genet Dev. 2015;35:119-25.

27. Blenda A, Fang DD, Rami J-F, Garsmeur O, Luo F, Lacape J-M. A high density consensus genetic map of tetraploid cotton that integrates multiple component maps through molecular marker redundancy check. PLoS One. 2012;7, e45739.

28. Korbecka G, Rymer PD, Harris SA, Pannell JR. Solving the problem of ambiguous paralogy for marker loci: microsatellite markers with diploid inheritance in allohexaploid Mercurialis annua (Euphorbiaceae). J Hered. 2010;101:504-11.

29. Konovalov FA, Goncharov NP, Goryunova S, Shaturova A, Proshlyakova T, Kudryavtsev A. Molecular markers based on LTR retrotransposons BARE-1 and Jeli uncover different strata of evolutionary relationships in diploid wheats. Mol Genet Genomics. 2010;283:551-63.

30. Metcalfe CJ, Oliveira SG, Gaiarsa JW, Aitken KS, Carneiro MS, Zatti F, et al. Using quantitative PCR with retrotransposon-based insertion polymorphisms as markers in sugarcane. J Exp Bot. 2015;66:4239-50.

31. Nybom H. Comparison of different nuclear DNA markers for estimating intraspecific genetic diversity in plants. Mol Ecol. 2004;13:1143-55.

32. Maguire TL, Peakall R, Saenger P. Comparative analysis of genetic diversity in the mangrove species Avicennia marina (Forsk.) Vierh. (Avicenniaceae) detected by AFLPs and SSRs. Theor Appl Genet. 2002;104:388-98.

33. Victoria FC, da Maia LC, de Oliveira AC. In silico comparative analysis of SSR markers in plants. BMC Plant Biol. 2011;11:15.

34. Schulman AH, Flavell A, Paux E, Ellis THN. The application of LTR retrotransposons as molecular markers in plants. Methods Mol Biol. 2012;859:115-53.

35. Garcia AAF, Mollinari M, Marconi TG, Serang OR, Silva RR, Vieira MLC, et al. SNP genotyping allows an in-depth characterisation of the genome of sugarcane and other complex autopolyploids. Sci Rep. 2013;3:3399.

36. Elshire RJ, Glaubitz JC, Sun Q, Poland JA, Kawamoto K, Buckler ES, et al. A robust, simple genotyping-by-sequencing (GBS) approach for high diversity species. PLoS One. 2011;6, e19379.

37. Byrne S, Czaban A, Studer B, Panitz F, Bendixen C, Asp T. Genome wide allele frequency fingerprints (GWAFFs) of populations via genotyping by sequencing. PLoS One. 2013;8, e57438.

38. Fjellheim S, Tanhuanpää P, Marum P, Manninen O, Rognli OA. Phenotypic or molecular diversity screening for conservation of genetic resources? An example from a genebank collection of the temperate forage grass timothy. Crop Sci. 2015;55:1646-59. 